KYUNGPOOK Math. J. 55(2015), 531-540

http://dx.doi.org/10.5666/KMJ.2015.55.3.531

pISSN 1225-6951 eISSN 0454-8124

(C) Kyungpook Mathematical Journal

\title{
Weyl Type Theorems for Unbounded Hyponormal Operators
}

\author{
Anuradha Gupta \\ Department of Mathematics, Delhi College of Arts and Commerce, University of \\ Delhi, Netaji Nagar, New Delhi-110023, India \\ e-mail : dishna2@yahoo.in \\ Karuna MamTANi* \\ Department of Mathematics, Faculty of Mathematical Sciences, University of Delhi, \\ New Delhi-11000\%, India \\ e-mail : karunamamtani@gmail.com
}

Abstract. If $\mathrm{T}$ is an unbounded hyponormal operator on an infinite dimensional complex Hilbert space H with $\rho(\mathrm{T}) \neq \phi$, then it is shown that T satisfies Weyl's theorem, generalized Weyl's theorem, Browder's theorem and generalized Browder's theorem. The equivalence of generalized Weyl's theorem with generalized Browder's theorem, property (gw) with property $(\mathrm{gb})$ and property $(\mathrm{w})$ with property (b) have also been established. It is also shown that a-Browder's theorem holds for $\mathrm{T}$ as well as its adjoint $\mathrm{T}^{*}$.

\section{Introduction}

Throughout this paper $H$ will be an infinite dimensional complex Hilbert space and $C(H)$ denotes the set of all closed linear operators from $H$ to $H$. By $\mathcal{D}(\mathrm{T})$, $\mathcal{R}(\mathrm{T})$ and $\mathcal{N}(\mathrm{T})$ we denote the domain, range and null space of $\mathrm{T}$, respectively. If the range of an operator $\mathrm{T} \in C(H)$ is closed and nullity of $\mathrm{T}, \alpha(\mathrm{T})=\operatorname{dim} \mathcal{N}(\mathrm{T})<\infty$ (respectively, defect of $\mathrm{T}, \beta(\mathrm{T})=\operatorname{codim} \mathcal{R}(\mathrm{T})<\infty$ ) then $\mathrm{T}$ is called an upper semiFredholm (respectively, lower semi-Fredholm) operator. A semi-Fredholm operator is an upper or lower semi-Fredholm operator. If both $\alpha(\mathrm{T})$ and $\beta(\mathrm{T})$ are finite then $\mathrm{T}$ is called a Fredholm operator. By $S F_{+}(H)$ (respectively, $S F_{-}(H)$ ) we denote the

\footnotetext{
* Corresponding Author.

Received October 2, 2014; revised November 16, 2014; accepted February 21, 2015.

2010 Mathematics Subject Classification: 47A53, 47B20.

Key words and phrases: Unbounded hyponormal operators, Weyl-type theorems, property (w), property (b).

The second author is supported by the Junior Research fellowship of Council of Scientific and Industrial Research, India, under the University Grants Commission Fellowship scheme (grant no. JRF/AA/139/F-211/2012-13).
} 
class of upper (respectively, lower) semi-Fredholm operators. For $\mathrm{T} \in S F_{+}(H) \cup$ $S F_{-}(H)$, index of $\mathrm{T}$ is defined as $\operatorname{ind}(\mathrm{T})=\alpha(\mathrm{T})-\beta(\mathrm{T})$. An operator $T \in C(H)$ is called Weyl if it is Fredholm of index 0 and the Weyl spectrum of $\mathrm{T}$ is defined as $\sigma_{W}(\mathrm{~T})=\{\lambda \in \mathbb{C}: \mathrm{T}-\lambda \mathrm{I}$ is not Weyl $\}$. Also we have

$$
\begin{aligned}
& S F_{+}^{-}(H)=\left\{T \in C(H): T \in S F_{+}(H) \text { and } \operatorname{ind}(T) \leqslant 0\right\}, \\
& S F_{-}^{+}(H)=\left\{T \in C(H): T \in S F_{-}(H) \text { and } \operatorname{ind}(T) \geqslant 0\right\}
\end{aligned}
$$

and these operators generate the following spectrum

$$
\begin{aligned}
\sigma_{S F_{+}^{-}}(T) & =\left\{\lambda \in \mathbb{C}: T-\lambda I \notin S F_{+}^{-}(H)\right\} \\
\sigma_{S F_{-}^{+}}(T) & =\left\{\lambda \in \mathbb{C}: T-\lambda I \notin S F_{-}^{+}(H)\right\} .
\end{aligned}
$$

The concept of Fredholm operators was generalized by Berkani [5] to the class of B-Fredholm operators in the following way: Let $\mathrm{T} \in C(H)$ and let

$$
\Delta(\mathrm{T})=\left\{n \in \mathbb{N}: \forall m \in \mathbb{N}, m \geqslant n \Rightarrow \mathcal{R}\left(\mathrm{T}^{n}\right) \cap \mathcal{N}(\mathrm{T}) \subseteq \mathcal{R}\left(\mathrm{T}^{m}\right) \cap \mathcal{N}(\mathrm{T})\right\} .
$$

Then the degree of stable iteration of $\mathrm{T}$ is defined as $\operatorname{dis}(\mathrm{T})=\inf \Delta(\mathrm{T})$ where $\operatorname{dis}(\mathrm{T})$ $=\infty$ if $\Delta(\mathrm{T})=\phi$. Let $\mathrm{T} \in C(H)$ be densely defined on $H$. We say that $\mathrm{T} \in C(H)$ is a semi B-Fredholm operator if it is either upper or lower semi B-Fredholm operator, where $\mathrm{T}$ is an upper (respectively, lower) semi B-Fredholm operator if there exists an integer $d \in \Delta(\mathrm{T})$ such that $\mathcal{R}\left(\mathrm{T}^{d}\right)$ is closed and $\operatorname{dim}\left\{\mathcal{N}(\mathrm{T}) \cap \mathcal{R}\left(\mathrm{T}^{d}\right)\right\}<\infty$ (respectively, codim $\left\{\mathcal{R}(\mathrm{T})+\mathcal{N}\left(\mathrm{T}^{d}\right)\right\}<\infty$ ).

In this case, index of $\mathrm{T}$ is defined as the number

$$
\operatorname{ind}(\mathrm{T})=\operatorname{dim}\left\{\mathcal{N}(\mathrm{T}) \cap \mathcal{R}\left(\mathrm{T}^{d}\right)\right\}-\operatorname{codim}\left\{\mathcal{R}(\mathrm{T})+\mathcal{N}\left(\mathrm{T}^{d}\right)\right\} .
$$

Also, we say that $\mathrm{T}$ is a B-Fredholm operator if $\mathrm{T}$ is both upper and lower semi B-Fredholm operator, that is, there exists an integer $d \in \Delta(\mathrm{T})$ such that $\mathrm{T}$ satisfies the following conditions:

(i) $\operatorname{dim}\left\{\mathcal{N}(\mathrm{T}) \cap \mathcal{R}\left(\mathrm{T}^{d}\right)\right\}<\infty$

(ii) $\operatorname{codim}\left\{\mathcal{R}(\mathrm{T})+\mathcal{N}\left(\mathrm{T}^{d}\right)\right\}<\infty$.

Let $S B F_{+}(H)$ denote the class of all upper semi B-Fredholm operators. Then

$$
\begin{aligned}
& S B F_{+}^{-}(H)=\left\{T \in C(H): T \in S B F_{+}(H) \text { and } \operatorname{ind}(T) \leqslant 0\right\} \quad \text { and } \\
& \sigma_{S B F_{+}^{-}}(H)=\left\{\lambda \in \mathbb{C}: T-\lambda I \notin S B F_{+}^{-}(H)\right\} .
\end{aligned}
$$

An operator $\mathrm{T} \in C(H)$ is said to be $B$-Weyl if it is a B-Fredholm operator of index zero and the $B$-Weyl spectrum of $\mathrm{T}$ is then defined as $\sigma_{B W}(\mathrm{~T})=\{\lambda \in \mathbb{C}: \mathrm{T}$ $-\lambda \mathrm{I}$ is not $\mathrm{B}-\mathrm{Weyl}\}$. 
The ascent $\mathrm{p}(\mathrm{T})$ and descent $\mathrm{q}(\mathrm{T})$ of an operator $\mathrm{T} \in C(H)$ are given by

$$
\begin{aligned}
& \mathrm{p}(\mathrm{T})=\inf \left\{n: \mathcal{N}\left(\mathrm{T}^{n}\right)=\mathcal{N}\left(\mathrm{T}^{n+1}\right)\right\} \quad \text { and } \\
& \mathrm{q}(\mathrm{T})=\inf \left\{n: \mathcal{R}\left(\mathrm{T}^{n}\right)=\mathcal{R}\left(\mathrm{T}^{n+1}\right)\right\} .
\end{aligned}
$$

An operator $\mathrm{T} \in C(H)$ is said to be upper semi-Browder (respectively, lower semi-Browder) if $\mathrm{T}$ is upper semi-Fredholm with $\mathrm{p}(\mathrm{T})<\infty$ (respectively, lower semi-Fredholm with $\mathrm{q}(\mathrm{T})<\infty)$. If $\mathrm{T}$ is both upper and lower semi-Browder, that is, if $\mathrm{T}$ is a Fredholm operator with ascent and descent both finite, then $\mathrm{T}$ is Browder. The upper-Browder, lower-Browder and Browder spectra are defined as

$$
\begin{aligned}
\sigma_{u b}(T) & =\{\lambda \in \mathbb{C}: T-\lambda I \text { not upper semi-Browder }\}, \\
\sigma_{l b}(T) & =\{\lambda \in \mathbb{C}: T-\lambda I \text { not lower semi-Browder }\} \text { and } \\
\sigma_{b}(T) & =\{\lambda \in \mathbb{C}: T-\lambda I \text { not Browder }\}, \text { respectively. }
\end{aligned}
$$

Clearly, $\sigma_{S F_{+}^{-}}(\mathrm{T}) \subseteq \sigma_{u b}(\mathrm{~T})$ and $\sigma_{w}(\mathrm{~T}) \subseteq \sigma_{b}(\mathrm{~T})$.

In [15], Weyl asserts that if $\mathrm{T}$ is a hermitian operator then $\sigma_{w}(T)$ consists precisely of all points in $\sigma(T)$ except the isolated eigenvalues of finite multiplicity. Weyl's theorem has been extended from hermitian operators to the class of bounded normal, hyponormal and Toeplitz operators [8]. Further in [3], Berkani proved that if $\mathrm{T}$ is a bounded normal operator acting on a Hilbert space $\mathrm{H}$, then $\sigma_{B W}(\mathrm{~T})=$ $\sigma(\mathrm{T}) \backslash \mathrm{E}(\mathrm{T})$, where $\mathrm{E}(\mathrm{T})$ is the set of all isolated eigenvalues of $\mathrm{T}$. This gives the generalization of the Weyl's theorem. Also, in [4] he proved this generalized version of classical Weyl's theorem for bounded hyponormal operators.

In this paper, we denote by $\Re(H)=\{\mathrm{T} \in C(H)$ : $\mathrm{T}$ is an unbounded hyponormal operator with resolvent set $\rho(\mathrm{T}) \neq \phi\}$ and we study several Weyl-type theorems and properties for operators $\mathrm{T} \in \Re(H)$. The second section deals with the spectrum of an unbounded hyponormal operator where we show that operators $\mathrm{T} \in \Re(H)$ are polaroid (every isolated spectral point is a pole) with ascent less than or equal to 1. In the third section, we show that T satisfies Weyl's theorem, generalized Weyl's theorem and Browder's theorem. Also, it is shown that a-Browder's theorem holds for $\mathrm{T}$ as well as its adjoint $\mathrm{T}^{*}$. In the fourth section, the results of section 2 are used to establish the equivalence of generalized Weyl's theorem with generalized Browder's theorem, Weyl's theorem with Browder's theorem and property (gw) ([2]) with property (gb) ([7]). Moreover, the two variants of Weyl's theorem, property (w) ([13]) and property (b) ([7]) are also shown to be equivalent. Finally, as a conclusion, we summarize the relations between Weyl-type theorems, Browder-type theorems and various properties in a diagram.

\section{Spectrum of an Unbounded Hyponormal Operator}

A linear operator $\mathrm{T}$ is called hyponormal if (i) $\mathrm{T}$ is closed, (ii) $\mathcal{D}(\mathrm{T})=\mathcal{D}\left(\mathrm{T}^{*}\right)$, $\mathcal{D}(\mathrm{T})$ is dense in $H$, and (iii) $\mathrm{T}^{*} \mathrm{~T}-\mathrm{TT}^{*} \geqslant 0$. Also, we have a characterization for unbounded hyponormal operators, namely, an operator $\mathrm{T}$ is hyponormal iff it 
satisfies (i) $\mathrm{T}^{*}$ exists, $\mathcal{D}(\mathrm{T})=\mathcal{D}\left(\mathrm{T}^{*}\right)$ and $\mathcal{D}(\mathrm{T})$ is dense in $H$ and (ii) $\|\mathrm{Tx}\| \geqslant\left\|\mathrm{T}^{*} \mathrm{x}\right\|$, for all $\mathrm{x} \in \mathcal{D}(\mathrm{T})=\mathcal{D}\left(\mathrm{T}^{*}\right)$.

Theorem 2.1. Let $T \in C(H)$ be an unbounded hyponormal operator. Then $p(\mathrm{~T}$ $\lambda \mathrm{I})=0$ or 1 for every $\lambda \in \mathbb{C}$.

Proof. Since $\mathrm{T}$ is hyponormal, so is $\mathrm{T}-\lambda \mathrm{I}$. Then for $x \in \mathcal{D}(\mathrm{T}-\lambda \mathrm{I})^{2}$, we have

$$
\begin{aligned}
\|(T-\lambda I) x\|^{2} & =|\langle(T-\lambda I) x,(T-\lambda I) x\rangle| \\
& =\left|\left\langle x,(T-\lambda I)^{*}(T-\lambda I) x\right\rangle\right| \\
& \leqslant\|x\|\left\|(T-\lambda I)^{*}(T-\lambda I) x\right\| \\
& \leqslant\|x\|\left\|(T-\lambda I)^{2} x\right\| .
\end{aligned}
$$

Therefore, $\|x\|\left\|(T-\lambda I)^{2} x\right\| \geqslant\|(T-\lambda I) x\|^{2}$, and hence $\mathcal{N}(T-\lambda \mathrm{I})^{2} \subseteq \mathcal{N}(\mathrm{T}-\lambda \mathrm{I})$. Since the reverse inclusion always holds, we have $\mathrm{p}(\mathrm{T}-\lambda \mathrm{I}) \leqslant 1$.

One of the interesting properties in Fredholm theory is the single valued extension property (SVEP). This property was first introduced by Dunford [9]. Mainly we concern with the localized version of SVEP, the SVEP at a point, introduced by Finch [10] and relate it to the finiteness of the ascent of an operator. Let $\mathrm{T}$ : $\mathcal{D}(\mathrm{T}) \subset H \rightarrow H$ be a closed linear mapping and let $\lambda_{o}$ be a complex number. The operator $\mathrm{T}$ has the single valued extension property (SVEP) at $\lambda_{o}$ if $f=0$ is the only solution to (T- $\lambda \mathrm{I}) f(\lambda)=0$ that is analytic in a neighborhood of $\lambda_{o}$. Also, T has SVEP if it has this property at every point $\lambda_{o}$ in the complex plane.

Theorem 2.2.([10]) Let $T \in C(H)$.

(i) If $p(\mathrm{~T}-\lambda \mathrm{I})$ is finite for some $\lambda \in \mathbb{C}$, then $T$ has SVEP at $\lambda$.

(ii) If $T$ is onto and not one-one, then $T$ does not have $S V E P$ at $\lambda=0$.

The second part of this theorem can also be rephrased as "If T has SVEP, then $\mathrm{T}$ is invertible whenever it is onto, that is, $\sigma(\mathrm{T})=\sigma_{s}(\mathrm{~T})$, where $\sigma_{s}(\mathrm{~T})$ is the surjective spectrum of T".

It is well known that the resolvent operator $\mathrm{R}_{\lambda}(\mathrm{T})=(\mathrm{T}-\lambda \mathrm{I})^{-1}$ is an analytic operator-valued function for all $\lambda \in \rho(\mathrm{T})$ and the isolated points of $\sigma(\mathrm{T})$ are either poles or essential singularities of $\mathrm{R}_{\lambda}(\mathrm{T})$. For $\mathrm{T} \in C(H)$, an isolated point $\lambda \in \sigma(\mathrm{T})$ is said to be a pole of order $\mathrm{p}$ if $\mathrm{p}=\mathrm{p}(\mathrm{T}-\lambda \mathrm{I})<\infty$ and $\mathrm{q}(\mathrm{T}-\lambda \mathrm{I})<\infty[12]$.

Theorem 2.3.([12]) Let $T$ be a closed linear operator with $\rho(\mathrm{T}) \neq \phi$. If $\lambda_{o} \in \sigma(\mathrm{T})$ and there exists two closed subspaces $M$ and $N$ such that $T-\lambda_{o} I$ is one-one mapping of $\mathcal{D}(\mathrm{T}) \cap M$ onto $M, T-\left.\lambda_{o} I\right|_{N}$ is nilpotent of order $p$ and $H=M \oplus N$, then $M=\mathcal{R}\left(T-\lambda_{o} I\right)^{p}, N=\mathcal{N}\left(T-\lambda_{o} I\right)^{p}$ and $\lambda_{o}$ is a pole of the resolvent $R_{\lambda}(\mathrm{T})$ of order $p$. The above condition is also necessary.

Lemma 2.4. If $T \in \Re(H)$, then $\lambda$ is an isolated point of $\sigma(T)$ iff $\lambda$ is a simple pole of the resolvent of $T$.

Proof. If $\lambda$ is a pole of resolvent of T then $\lambda$ is an isolated point of $\sigma(T)$. 
Conversely, suppose $\lambda$ is an isolated point of $\sigma(T)$. Then $\{\lambda\}$ is a spectral set of $\mathrm{T}$ and by $[14, \S \mathrm{V} .10]$, there is a corresponding spectral projection operator $E_{o}$ such that $H=\mathcal{N}\left(E_{o}\right) \oplus \mathcal{R}\left(E_{o}\right)=\mathrm{X}_{1} \oplus \mathrm{X}_{2}$, say. We have, $\mathrm{T}$ is completely reduced by the pair of closed subspaces $\mathrm{X}_{1}$ and $\mathrm{X}_{2}$ and if $T_{1}=\left.T\right|_{X_{1}}$ and $T_{2}=\left.T\right|_{X_{2}}$, then $\sigma\left(T_{1}\right)=\sigma(T) \backslash\{\lambda\}$ and $\sigma\left(T_{2}\right)=\{\lambda\}$ so that $T-\lambda I$ is a one-one mapping of $\mathrm{X}_{1}$ onto itself.

$$
\begin{aligned}
& \text { Also, } \quad \mathcal{R}(T-\lambda I)=X_{1} \oplus(T-\lambda I) X_{2} \\
& =X_{1} \oplus 0 \quad(\because \mathrm{p}(\mathrm{T}-\lambda \mathrm{I}) \leqslant 1 \text { by Theorem } 2.1) . \\
& =X_{1} \oplus 0=\mathcal{R}(T-\lambda I) .
\end{aligned}
$$

Thus, $\mathrm{q}(\mathrm{T}-\lambda \mathrm{I})=\mathrm{p}(\mathrm{T}-\lambda \mathrm{I}) \leqslant 1$ and hence $\lambda$ is a simple pole of the resolvent operator $R_{\lambda}(T)$.

Let $\sigma_{a}(\mathrm{~T})$ be the approximate point spectrum of $\mathrm{T}$. By $\mathrm{E}(\mathrm{T})$ and $\mathrm{E}_{o}(\mathrm{~T})$ we denote the set of all isolated eigenvalues of $\mathrm{T}$ and the set of all isolated eigenvalues of finite multiplicities in $\sigma(\mathrm{T})$, respectively. Also, let $\pi(\mathrm{T})$ and $\pi_{o}(\mathrm{~T})$ denote the set of all poles and the set of all poles of finite multiplicities of the resolvent of $\mathrm{T}$, respectively. We say that $\mathrm{T} \in C(H)$ satisfies:

(i) Weyl's theorem if $\sigma(\mathrm{T}) \backslash \sigma_{w}(\mathrm{~T})=\mathrm{E}_{o}(\mathrm{~T})$.

(ii) Generalized Weyl's theorem if $\sigma(\mathrm{T}) \backslash \sigma_{B W}(\mathrm{~T})=\mathrm{E}(\mathrm{T})$.

(iii) Browder's theorem if $\sigma_{w}(\mathrm{~T})=\sigma_{b}(\mathrm{~T})$.

(iv) Generalized Browder's theorem if $\sigma(\mathrm{T}) \backslash \sigma_{B W}(\mathrm{~T})=\pi(\mathrm{T})$.

(v) a-Browder's theorem if $\sigma_{S F_{+}^{-}}(\mathrm{T})=\sigma_{u b}(\mathrm{~T})$.

(vi) property (w) if $\sigma_{a}(\mathrm{~T}) \backslash \sigma_{S F_{+}^{-}}(\mathrm{T})=\mathrm{E}_{o}(\mathrm{~T})$.

(vii) property (gw) if $\sigma_{a}(\mathrm{~T}) \backslash \sigma_{S B F_{+}^{-}}(\mathrm{T})=\mathrm{E}(\mathrm{T})$.

(viii) property (b) if $\sigma_{a}(\mathrm{~T}) \backslash \sigma_{S F_{+}^{-}}(\mathrm{T})=\sigma(\mathrm{T}) \backslash \sigma_{b}(\mathrm{~T})$.

(ix) property (gb) if $\sigma_{a}(\mathrm{~T}) \backslash \sigma_{S B F_{+}^{-}}(\mathrm{T})=\pi(\mathrm{T})$.

\section{Weyl-Type Theorems}

In this section we prove several Weyl-type theorems for $\mathrm{T} \in \Re(H)$.

Theorem 3.1. If $T \in \Re(H)$, then Weyl's theorem holds for $T$.

Proof. Let $\lambda \in \sigma(\mathrm{T}) \backslash \sigma_{w}(\mathrm{~T})$. Then $\mathcal{R}(\mathrm{T}-\lambda \mathrm{I})$ is closed and $\alpha(\mathrm{T}-\lambda \mathrm{I})=\beta(\mathrm{T}-\lambda \mathrm{I})$ $<\infty$. If $\alpha(\mathrm{T}-\lambda \mathrm{I})=\beta(\mathrm{T}-\lambda \mathrm{I})=0, \mathrm{~T}-\lambda \mathrm{I}$ is a one-one mapping of $\mathcal{D}(\mathrm{T})$ onto all of $H$. The inverse $(\mathrm{T}-\lambda \mathrm{I})^{-1}$ is then closed and hence bounded, thus $\lambda \notin \sigma(\mathrm{T})$, 
which is a contradiction. Hence, $\alpha(\mathrm{T}-\lambda \mathrm{I})>0$. Also, since $\mathrm{p}(\mathrm{T}-\lambda \mathrm{I})=1<\infty,[1$, Theorem 3.4(iv)] gives $\mathrm{q}(\mathrm{T}-\lambda \mathrm{I})=\mathrm{p}(\mathrm{T}-\lambda \mathrm{I})<\infty$. Therefore, $\lambda \in \mathrm{E}_{o}(\mathrm{~T})$.

Conversely, suppose $\lambda \in \mathrm{E}_{o}(\mathrm{~T})$. Then $\lambda$ is a pole of order $\mathrm{p}(\mathrm{T}-\lambda \mathrm{I})=1$ so that $H=\mathcal{R}(\mathrm{T}-\lambda \mathrm{I}) \oplus \mathcal{N}(\mathrm{T}-\lambda \mathrm{I})$. Therefore, $\beta(\mathrm{T}-\lambda \mathrm{I})=\alpha(\mathrm{T}-\lambda \mathrm{I})<\infty$ and hence, $\lambda \in \sigma(\mathrm{T}) \backslash \sigma_{w}(\mathrm{~T})$. Thus, Weyl's theorem holds for $\mathrm{T}$.

Theorem 3.2. If $T \in \Re(H)$, then $T$ satisfies generalized Weyl's theorem.

Proof. Suppose $\lambda \in \sigma(\mathrm{T}) \backslash \sigma_{B W}(\mathrm{~T})$. Then $\mathrm{T}-\lambda \mathrm{I}$ is a B-Fredholm operator of index 0 . By [5, theorem 2.4], there exist two closed invariant subspaces $M$ and $N$ of $H$ such that $H=M \oplus N, \mathrm{~T}_{o}=\left.(\mathrm{T}-\lambda \mathrm{I})\right|_{M}$ is a closed Fredholm operator of index 0 , and $\mathrm{T}_{1}=\left.(\mathrm{T}-\lambda \mathrm{I})\right|_{N}$ is a nilpotent operator. Since $\mathrm{T}$ is hyponormal and $M$ is a closed invariant subspace of $\mathrm{T}$, so $\mathrm{S}=\left.\mathrm{T}\right|_{M}$ is a hyponormal operator. Also ( $\mathrm{S}$ $-\lambda \mathrm{I})\left.\right|_{M}=\mathrm{T}_{o}$ is a Fredholm operator of index 0 so that $\lambda \notin \sigma_{w}(\mathrm{~S})$. We have the following two cases:

Case (i): $\lambda \in \sigma(\mathrm{S})$.

$\mathrm{S}$ being hyponormal satisfies Weyl's theorem and $\lambda \notin \sigma_{w}(\mathrm{~S})$, therefore $\lambda \epsilon$ $\mathrm{E}_{o}(\mathrm{~S})$. In particular, $\lambda$ is isolated in $\sigma(\mathrm{S})$ or 0 is isolated in $\sigma\left(\mathrm{T}_{o}\right)$. Since $\mathrm{T}-\lambda \mathrm{I}$ $=\mathrm{T}_{o} \oplus \mathrm{T}_{1}$ and $\mathrm{T}_{1}$ is a nilpotent operator, so $\sigma\left(\mathrm{T}_{o}\right) \backslash\{0\}=\sigma(\mathrm{T}-\lambda \mathrm{I}) \backslash\{0\}$. Therefore, 0 is isolated in $\sigma(\mathrm{T}-\lambda \mathrm{I})$ or equivalently, $\lambda$ is isolated in $\sigma(\mathrm{T})$. Also $\lambda$, being an eigenvalue of $\mathrm{S}$, is an eigenvalue of $\mathrm{T}$. Hence, $\lambda \in \mathrm{E}(\mathrm{T})$.

Case (ii): $\lambda \notin \sigma(\mathrm{S})$.

In this case, $\mathrm{T}-\lambda \mathrm{I}=\mathrm{T}_{o} \oplus \mathrm{T}_{1}=\left.(\mathrm{S}-\lambda \mathrm{I})\right|_{M} \oplus \mathrm{T}_{1}$ where $\left.(\mathrm{S}-\lambda \mathrm{I})\right|_{M}$ is a one-one mapping of $\mathcal{D}(\mathrm{T}) \cap M$ onto $M$ and $\mathrm{T}_{1}$ is a nilpotent operator. Therefore, $\lambda$ is a pole of the resolvent operator $\mathrm{R}_{\lambda}(\mathrm{T})$. Hence, $\lambda$ is an isolated eigenvalue of $\mathrm{T}$ so that $\lambda \in \mathrm{E}(\mathrm{T})$.

Therefore, $\sigma(\mathrm{T}) \backslash \sigma_{B W}(\mathrm{~T}) \subseteq \mathrm{E}(\mathrm{T})$.

Conversely, let $\lambda \in \mathrm{E}(\mathrm{T})$. Then, $\alpha(\mathrm{T}-\lambda \mathrm{I})>0$ and $\mathrm{p}(\mathrm{T}-\lambda \mathrm{I})=1 . \quad \lambda$ being an isolated point is a pole of order $\mathrm{p}(\mathrm{T}-\lambda \mathrm{I})=1$. Thus, $H=\mathcal{R}(\mathrm{T}-\lambda \mathrm{I}) \oplus \mathcal{N}(\mathrm{T}-$ $\lambda \mathrm{I})=M \oplus N$, say. Now, $\left.(\mathrm{T}-\lambda \mathrm{I})\right|_{M}$ is a one-one operator from $\mathcal{D}(\mathrm{T}) \cap M$ onto $M$, and hence a closed Fredholm operator of index 0 , and $\left.(\mathrm{T}-\lambda \mathrm{I})\right|_{N}$ is a nilpotent operator of index 1. By [5, Theorem 2.4], $\mathrm{T}-\lambda \mathrm{I}$ is a B-Fredholm operator of index 0 . Therefore, $\lambda \in \sigma(\mathrm{T}) \backslash \sigma_{B W}(\mathrm{~T})$.

Theorem 3.3. Every unbounded hyponormal operator satisfies Browder's theorem. Proof. Since for every unbounded hyponormal operator T we have $\mathrm{p}(\mathrm{T}-\lambda \mathrm{I})<\infty$, Browder's theorem follows from [1, Theorem 3.4].

Theorem 3.4. If $T$ is an unbounded hyponormal operator, then $T$ and $T^{*}$ satisfy a-Browder's theorem.

Proof. Suppose that $\lambda \notin \sigma_{u b}(\mathrm{~T})$. Then $\mathrm{T}-\lambda \mathrm{I}$ is upper semi-Fredholm operator and $\mathrm{p}(\mathrm{T}-\lambda \mathrm{I})<\infty,\left[1\right.$, Theorem 3.4(i)] gives $\operatorname{ind}(\mathrm{T}-\lambda \mathrm{I}) \leqslant 0$ so that $\lambda \notin \sigma_{S F_{+}^{-}}(\mathrm{T})$. Thus, $\sigma_{S F_{+}^{-}}(\mathrm{T}) \subseteq \sigma_{u b}(\mathrm{~T})$.

Conversely, since $\mathrm{p}(\mathrm{T}-\lambda \mathrm{I})<\infty$ for all $\lambda \in \mathbb{C}$, so $\sigma_{u b}(\mathrm{~T}) \subseteq \sigma_{S F_{+}^{-}}(\mathrm{T})$ and hence $\mathrm{T}$ satisfies a-Browder's theorem. 
Now suppose $\lambda \notin \sigma_{S F_{+}^{-}}\left(\mathrm{T}^{*}\right)=\sigma_{S F_{-}^{+}}(\mathrm{T})$. Then $\mathrm{T}-\lambda \mathrm{I}$ is lower semi-Fredholm with $\operatorname{ind}(\mathrm{T}-\lambda \mathrm{I}) \geqslant 0$. Since $\mathrm{T}-\lambda \mathrm{I}$ is hyponormal, we have $\mathrm{p}(\mathrm{T}-\lambda \mathrm{I})<\infty$ so that by $[1$, Theorem 3.4(i)], ind $(\mathrm{T}-\lambda \mathrm{I}) \leqslant 0$. Thus, ind $(\mathrm{T}-\lambda \mathrm{I})=0$ and hence, $\mathrm{q}(\mathrm{T}-$ $\lambda \mathrm{I})=\mathrm{p}(\mathrm{T}-\lambda \mathrm{I})<\infty$. Therefore, $\lambda \notin \sigma_{l b}(\mathrm{~T})=\sigma_{u b}\left(\mathrm{~T}^{*}\right)$. Since the reverse inclusion holds for every operator, we have that $\mathrm{T}^{*}$ satisfies a-Browder's theorem.

The following example of an operator $\mathrm{T} \in \Re(H)$ illustrates all the above theorems:

Example 3.5. Let $H=l^{2}$ and let $\mathrm{T}$ be defined as:

$$
\begin{aligned}
T\left(x_{1}, x_{2}, x_{3}, \ldots\right) & =\left(0, x_{1}, 2 x_{2}, 3 x_{3}, 4 x_{4}, \ldots\right) \\
& =\left(0, a_{1} x_{1}, a_{2} x_{2}, a_{3} x_{3}, a_{4} x_{4}, \ldots\right)
\end{aligned}
$$

where, $a_{n}=n$ for all $n \in \mathbb{N}$ and $\mathcal{D}(\mathrm{T})=\left\{\left(x_{n}\right) \in l^{2}: \sum_{j=1}^{\infty}\left|a_{j} x_{j}\right|^{2}<\infty\right\}$.

If $c_{o o}=\left\{x=\left(x_{n}\right): x_{n} \neq 0\right.$ for only finitely many $\left.n \in \mathbb{N}\right\}$, then $c_{o o}$ is dense in $l^{2}$. Since $c_{o o} \subseteq \mathcal{D}(\mathrm{T})$, so that $\mathcal{D}(\mathrm{T})$ is dense in $H$. Also, since $\left|a_{n}\right| \leqslant\left|a_{n+1}\right|$, T is an unbounded hyponormal operator.

Consider an operator $\mathrm{S}$ on $H$ defined as:

$$
\begin{aligned}
S\left(x_{1}, x_{2}, x_{3}, \ldots\right) & =\left(x_{2}, 2 x_{3}, 3 x_{4}, 4 x_{5}, \ldots\right) \\
& =\left(\overline{a_{1}} x_{2}, \overline{a_{2}} x_{3}, \overline{a_{3}} x_{4}, \overline{a_{4}} x_{5}, \ldots\right)
\end{aligned}
$$

with $\mathcal{D}(\mathrm{S})=\left\{\left(x_{n}\right) \in l^{2}: \sum_{j=1}^{\infty}\left|\overline{a_{j}} x_{j}\right|^{2}<\infty\right\}$. Then $\mathrm{S}=\mathrm{T}^{*}$, the adjoint of $\mathrm{T}$.

Clearly, $\sigma_{p}(\mathrm{~T})=\phi$. Therefore, $\mathrm{E}(\mathrm{T})=\mathrm{E}_{o}(\mathrm{~T})=\phi$.

From [11], since $M=\lim _{n \rightarrow \infty}\left|a_{n}\right|=\infty$, we have

$\sigma(\mathrm{T})=\{\lambda:|\lambda| \leqslant M\}$ i.e. $\sigma(\mathrm{T})=\mathbb{C} \cup\{\infty\}$, the extended complex plane,

$\sigma_{a}(\mathrm{~T})=\{\lambda:|\lambda|=M\}$ i.e. $\sigma_{a}(\mathrm{~T})=\{\infty\}$ and

$\sigma_{p}\left(\mathrm{~T}^{*}\right)=\{\lambda:|\lambda|<M\}$ i.e. $\sigma_{p}\left(\mathrm{~T}^{*}\right)=\mathbb{C}$.

Case (i): $\lambda \in \sigma(\mathrm{T}), \lambda \neq \infty$.

Then, $\lambda \notin \sigma_{a}(\mathrm{~T})$ implies that $\alpha(\mathrm{T}-\lambda \mathrm{I})=0$, hence $\mathrm{p}(\mathrm{T}-\lambda \mathrm{I})=0$, and $\mathcal{R}(\mathrm{T}$ $\lambda \mathrm{I})$ is closed so that $\lambda \notin \sigma_{S F_{+}^{-}}(\mathrm{T})$ and $\lambda \notin \sigma_{u b}(\mathrm{~T})$. Also, $\lambda \in \sigma_{p}\left(\mathrm{~T}^{*}\right)$ implies that $\beta(\mathrm{T}-\lambda \mathrm{I})=\alpha\left(\mathrm{T}^{*}-\lambda \mathrm{I}\right) \neq 0$ so that $i n d(\mathrm{~T}-\lambda \mathrm{I}) \neq 0$ and hence $\lambda \in \sigma_{w}(\mathrm{~T}) \subseteq \sigma_{b}(\mathrm{~T})$.

Further, $\alpha(\mathrm{T}-\lambda \mathrm{I})=0$ implies that $\operatorname{dim}\left\{\mathcal{N}(\mathrm{T}-\lambda \mathrm{I}) \cap \mathcal{R}(\mathrm{T}-\lambda \mathrm{I})^{d}\right\}=0$. Also $\mathrm{p}(\mathrm{T}-\lambda \mathrm{I})=0$ implies that $\operatorname{codim}\left\{\mathcal{R}(\mathrm{T}-\lambda \mathrm{I})+\mathcal{N}(\mathrm{T}-\lambda \mathrm{I})^{d}\right\}=\operatorname{codim} \mathcal{R}(\mathrm{T}-\lambda \mathrm{I})=$ $\beta(\mathrm{T}-\lambda \mathrm{I}) \neq 0$. Thus B-Fredholm index of $\mathrm{T}-\lambda \mathrm{I}$ is non-zero and hence $\lambda \in \sigma_{B W}(\mathrm{~T})$.

Case (ii): $\lambda=\infty$. 
Then, $\lambda \notin \sigma_{p}(\mathrm{~T})$ and $\lambda \in \sigma_{a}(\mathrm{~T})$ implies that $\alpha(\mathrm{T}-\lambda \mathrm{I})=0$ but $\mathcal{R}(\mathrm{T}-\lambda \mathrm{I})$ is not closed. Hence, $\lambda=\infty$ belongs to $\sigma_{S F_{+}^{-}}(\mathrm{T}), \sigma_{u b}(\mathrm{~T}), \sigma_{w}(\mathrm{~T})$ and $\sigma_{b}(\mathrm{~T})$.

Also, $\operatorname{codim}\left\{\mathcal{R}(\mathrm{T}-\lambda \mathrm{I})+\mathcal{N}(\mathrm{T}-\lambda \mathrm{I})^{d}\right\}=\operatorname{codim} \mathcal{R}(\mathrm{T}-\lambda \mathrm{I})=\beta(\mathrm{T}-\lambda \mathrm{I}) \nless \infty$ (because otherwise $\mathcal{R}(\mathrm{T}-\lambda \mathrm{I})$ must be closed). Hence, $\lambda \in \sigma_{B W}(\mathrm{~T})$.

From the above cases, we have

$\sigma_{w}(\mathrm{~T})=\mathbb{C} \cup\{\infty\}=\sigma_{b}(\mathrm{~T})$ and hence $\mathrm{T}$ satisfies Browder's theorem,

$\sigma_{S F_{+}^{-}}(\mathrm{T})=\{\infty\}=\sigma_{u b}(\mathrm{~T})$ and hence $\mathrm{T}$ satisfies a-Browder's theorem,

$\sigma(\mathrm{T}) \backslash \sigma_{w}(\mathrm{~T})=\phi=\mathrm{E}_{o}(\mathrm{~T})$ and hence $\mathrm{T}$ satisfies Weyl's theorem, and

$\sigma(\mathrm{T}) \backslash \sigma_{B W}(\mathrm{~T})=\phi=\mathrm{E}(\mathrm{T})$ and hence T satisfies generalized Weyl's theorem.

\section{Equivalent Variants of Weyl-Type Theorems and Hyponormal Oper- ators}

It is known that property $(\mathrm{gw})$ implies property $(\mathrm{gb})$ and property $(\mathrm{w})$ implies property (b) for every $\mathrm{T} \in B(H)$, but the converses of these results do not hold true in general [7]. In this section we prove the equivalence of property (gw) with (gb) and property (w) with (b) for an operator $\mathrm{T} \in \Re(H)$. Further, we also prove that for such a T, generalized Weyl's theorem is equivalent to generalized Browder's theorem and Weyl's theorem is equivalent to Browder's theorem.

Theorem 4.1. Let $T \in \Re(H)$. Then property (b) holds for $T$ iff property (w) holds for $T$.

Proof. Suppose T satisfies property (b).

Let $\lambda \in \sigma_{a}(\mathrm{~T}) \backslash \sigma_{S F_{+}^{-}}(\mathrm{T})$. Since $\mathrm{T}$ satisfies property (b), $\lambda \in \sigma(\mathrm{T}) \backslash \sigma_{b}(\mathrm{~T})$ so that $\mathrm{T}-\lambda \mathrm{I}$ is a Fredholm operator of index $0, \mathrm{p}(\mathrm{T}-\lambda \mathrm{I})<\infty$ and $\mathrm{q}(\mathrm{T}-\lambda \mathrm{I})<\infty$. Then, $\lambda$ is an isolated point of $\sigma(\mathrm{T})$. Also $0<\alpha(\mathrm{T}-\lambda \mathrm{I})<\infty$ (otherwise $\alpha(\mathrm{T}-\lambda \mathrm{I}$ ) $=\beta(\mathrm{T}-\lambda \mathrm{I})=0$ and $\lambda \notin \sigma(\mathrm{T}))$. Therefore, $\lambda \in \mathrm{E}_{o}(\mathrm{~T})$.

Conversely, suppose $\lambda \notin \sigma_{a}(\mathrm{~T}) \backslash \sigma_{S F_{+}^{-}}(\mathrm{T})$. Since $\mathrm{T}$ is hyponormal, $\mathrm{p}(\mathrm{T}-\lambda \mathrm{I})$ $<\infty$. By [1, Theorem 3.4(i)], ind $(\mathrm{T}-\lambda \mathrm{I})^{+} \leqslant 0$. We have to show that $\lambda \notin \mathrm{E}_{o}(\mathrm{~T})$. We have the following two cases:

Case (i): $\alpha(\mathrm{T}-\lambda \mathrm{I})=\infty$.

Then $\lambda \notin \mathrm{E}_{o}(\mathrm{~T})$.

Case (ii): $\alpha(\mathrm{T}-\lambda \mathrm{I})<\infty$.

Since $\mathrm{T}-\lambda \mathrm{I}$ is not upper semi-Fredholm operator, therefore, $\mathcal{R}(\mathrm{T}-\lambda \mathrm{I})$ is not closed. If $\lambda \in \mathrm{E}_{o}(\mathrm{~T})$, then $\lambda$ is a pole of order $\mathrm{p}(\mathrm{T}-\lambda \mathrm{I})=1$. Then, $H=\mathcal{R}(\mathrm{T}-\lambda \mathrm{I})$ $\oplus \mathcal{N}(\mathrm{T}-\lambda \mathrm{I})$ so that $\mathcal{R}(\mathrm{T}-\lambda \mathrm{I})$ is closed which is a contradiction. Therefore, $\lambda \notin$ $\mathrm{E}_{o}(\mathrm{~T})$.

Thus, $\mathrm{E}_{o}(\mathrm{~T}) \subseteq \sigma_{a}(\mathrm{~T}) \backslash \sigma_{S F_{+}^{-}}(\mathrm{T})$ and hence $\mathrm{T}$ satisfies property (w).

Conversely, suppose $\mathrm{T}$ satisfies property $(\mathrm{w})$.

Let $\lambda \in \sigma(\mathrm{T}) \backslash \sigma_{b}(\mathrm{~T})$. By definition, $\sigma(\mathrm{T}) \backslash \sigma_{b}(\mathrm{~T}) \subseteq \mathrm{E}_{o}(\mathrm{~T})$. Since $\mathrm{T}$ satisfies property $(\mathrm{w}), \lambda \in \mathrm{E}_{o}(\mathrm{~T})=\sigma(\mathrm{T}) \backslash \sigma_{S F_{+}^{-}}(\mathrm{T})$. 
Conversely, suppose $\lambda \in \sigma_{a}(\mathrm{~T}) \backslash \sigma_{S F_{+}^{-}}(\mathrm{T})$. Since $\mathrm{T}$ satisfies property (w), $\lambda \in$ $\mathrm{E}_{o}(\mathrm{~T})$. Then, $\lambda$ is a pole of order $\mathrm{p}(\mathrm{T}-\lambda \mathrm{I})=1$ so that $H=\mathcal{R}(\mathrm{T}-\lambda \mathrm{I}) \oplus \mathcal{N}(\mathrm{T}-\lambda \mathrm{I})$ and hence $\beta(\mathrm{T}-\lambda \mathrm{I})=\alpha(\mathrm{T}-\lambda \mathrm{I})<\infty$. By $[1$, Theorem 3.4(iv)], $\mathrm{q}(\mathrm{T}-\lambda \mathrm{I})=\mathrm{p}(\mathrm{T}-$ $\lambda \mathrm{I})=1$. Therefore, $\lambda \in \sigma(\mathrm{T}) \backslash \sigma_{b}(\mathrm{~T})$ so that $\mathrm{T}$ satisfies property (b).

The following theorem is a direct consequence of Lemma 2.4.

Theorem 4.2. For every $T \in \Re(H), E(\mathrm{~T})=\pi(\mathrm{T})$. In particular, $E_{o}(\mathrm{~T})=\pi_{o}(\mathrm{~T})$.

Remark 4.3. Since $\sigma(\mathrm{T}) \backslash \sigma_{b}(\mathrm{~T})=\pi_{o}(\mathrm{~T})$ for every $\mathrm{T} \in C(H)$, the above theorem implies that Weyl's theorem is equivalent to Browder's theorem for every $\mathrm{T} \in \Re(H)$. Now, Theorem 3.3 can be viewed as a corollary to Theorem 3.1.

Corollary 4.4. Let $T \in \Re(H)$. Then:

(i) generalized Weyl's theorem is equivalent to generalized Browder's theorem

(ii) property ( $\mathrm{gw})$ is equivalent to property ( $\mathrm{gb})$.

Remark 4.5. By above corollary and Theorem 3.2, we get that every $\mathrm{T} \in \Re(H)$ satisfies generalized Browder's theorem.

\section{Conclusion}

To summarize, we use the abbreviations $\mathrm{gW}, \mathrm{W},(\mathrm{gw})$ and $(\mathrm{w})$ to signify that an operator $\mathrm{T} \in \Re(H)$ satisfies generalized Weyl's theorem, Weyl's theorem, property $(\mathrm{gw})$, property $(\mathrm{w})$, respectively. Analogous abbreviations $\mathrm{aB}, \mathrm{gB}, \mathrm{B},(\mathrm{gb})$ and (b) have been used with respect to a-Browder's theorem, generalized Browder's theorem, Browder's theorem, property ( $\mathrm{gb}$ ) and property (b), respectively.

The following diagram shows the relations between Weyl-type theorems, Browder-type theorems and properties for an operator $\mathrm{T} \in \Re(H)$. The arrows signify implications between the theorems and properties. The numbers near the arrows are references to the results proved in the present paper. We notice that several one-sided implications that hold for an operator $\mathrm{T} \in B(H)$ become equivalences when we consider $\mathrm{T} \in \Re(H)$.

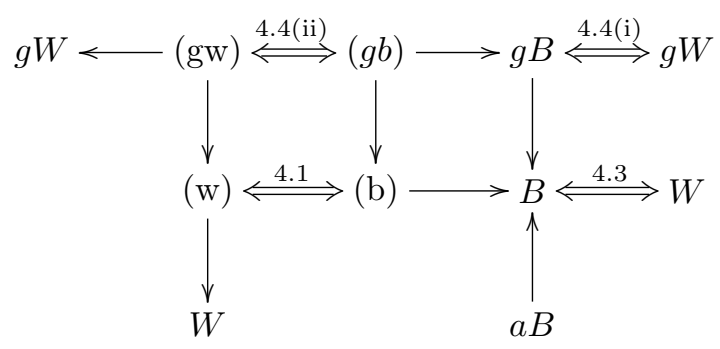

Acknowledgements. The authors would like to thank the referees for their valuable comments and suggestions. 


\section{References}

[1] P. Aiena, Fredholm and local spectral theory with applications to multipliers, Kluwer Acad. Publishers, (2004).

[2] M. Amouch, M. Berkani, On the property (gw), Mediterr. J. Math., 5(2008), 371-378.

[3] M. Berkani, Index of B-Fredholm operators and generalization of a Weyl theorem, Proc. Amer. Math. Soc., 130(2002), 1717-1723.

[4] M. Berkani, A. Arroud, Generalized Weyl's theorem and hyponormal operators, J. Aust. Math. Soc., 76(2)(2004), 291-302.

[5] M. Berkani, N. Castro-González, Unbounded B-Fredholm operators on Hilbert spaces, Proc. of the Edinburg Math. Soc., 51(2008), 285-296.

[6] M. Berkani, J. J. Koliha, Weyl type theorems for bounded linear operators, Acta Sci. Math. (Szeged), 69(2003), 359-376.

[7] M. Berkani, H. Zariouh, Extended Weyl type theorems, Mathematica Bohemica, 134(4)(2009), 369-378.

[8] L. A. Coburn, Weyl's theorem for nonnormal operators, The Michigan Mathematical Journal, 13(3)(1966), 285-288.

[9] N. Dunford, Spectral theory I. Resolution of the identity, Pacific Journal of Math., 2(1952), 559-614.

[10] J. K. Finch, The Single Valued Extension Property on a Banach Space, Pacific Journal of Math., 58 1(1975), 61-69.

[11] W. Gongbao, M. Jipu, Spectral characterization of Hyponormal Weighted Shifts, arXiv preprint math/0302275, (2003).

[12] D. C. Lay, Spectral Analysis Using Ascent, Descent, Nullity and Defect, Math. Ann., 184(1970), 197-214.

[13] V. Rakočević, On a class of operators, Mat. Vesnik., 37(1985), 423-426.

[14] A. E. Taylor, D. C. Lay Introduction to Functional Analysis, Second Edition, New York, Wiley and sons, (1980).

[15] H. Weyl, Über beschränkte quadatische Formen, deren Differenz Vollstetig ist, Rend. Circ. Mat. Palermo, 27(1909) 373-392. 